MAGNETOHYDRODYNAMICS Vol. 54 (2018), No. 1-2, pp. 23-26

DOI: $10.22364 / \mathrm{mhd} .54 .1-2.4$

\title{
NON-LINEAR WAVES IN A THIN LAYER OF MAGNETIC FLUID
}

\author{
A.N. Golubiatnikov \\ Lomonosov Moscow State University, 1 Michurinsky pr., 119192 Moscow, Russia
}

The problem of propagation of non-linear waves with a small amplitude in a thin layer of paramagnetic fluid with variable thickness in magnetic field is considered in shallow water theory. The equations of motion are derived and the method of solving the problem with weak discontinuity is developed. With the help of this method the problem with a weak shock wave is solved. An example of equilibrium initial state is considered.

1. Shallow water equations in magnetic field. The flat problem is considered. Let $y=\zeta(x, t)$ and $y=-h(x, t)$ be the forms of higher and lower surfaces in relation to the constant gravity field $g$. The function $h(x, t)$ is given. As known [1,2], equations of motion in a shallow water theory approximation could be derived in the assumption of smallness of the ratio of the layer thickness to the characteristic wavelength $\varepsilon=(\zeta+h) / \lambda \ll 1$. The permeability $\mu$ is considered to be piecewise constant, $\mu=1$ outside the layer and $\mu>1$ within the layer. Thus, the motion of fluid and magnetic field are related to each other only by the boundary conditions on the layer surface [3].

Vertically we solve the equation of equilibrium, and the solution defines the pressure

$$
p=p_{1}(x, t)-\rho g(y-\zeta) .
$$

Taking into account the order terms of $\varepsilon, \mathbf{n}=\left(-\zeta_{x}, 1\right)$ is normal to the surface of the layer, the tangent vector is $\tau=\left(1, \zeta_{x}\right)$. The subscript $x$ means a derivative.

The pressure above the layer is assumed to be zero. Then an arbitrary function $p_{1}$, considering the conditions $\left[H_{\tau}\right]=0$ and $\left[\mu H_{n}\right]=0$, is defined by the jump of the normal-normal component of the Maxwell stress tensor

$$
p_{1}=\frac{1}{8 \pi}\left[\mu\left(H_{n}^{2}-H_{\tau}^{2}\right)\right]_{0}^{1}=-\frac{\mu-1}{8 \pi \mu}\left(\left(H_{n}^{0}\right)^{2}+\mu\left(H_{\tau}^{0}\right)^{2}\right)<0 .
$$

The state outside the layer is denoted by zero, and the state into the layer is denoted by unity. Surface tension is not considered. The behaviour analysis of the magnetic field that is regular in the neighborhood of the layer shows that an external field distortion by the thin layer appears only in terms of the order $\varepsilon^{2}$. It could be observed in an example of flattened ellipsoid in [4]. Thus, in expression (2) calculated with the terms of order $\varepsilon$ only the external magnetic field is considered. Let us assume the term of the order of unity

$$
p_{1}=\frac{1}{8 \pi}\left([1 / \mu]\left(H_{2}^{0}\right)^{2}-[\mu]\left(H_{1}^{0}\right)^{2}\right) .
$$

A traditional approach applied to calculate hydrodynamics terms in the theory of an ideal incompressible homogeneous fluid [1,2], considering the known variable depth $h(x, t)$, gives the following equations for the average layer thickness at the longitudinal motion velocity $v$ and layer thickness $\zeta+h$

$$
(\zeta+h)_{t}+((\zeta+h) v)_{x}=0, \quad v_{t}+v v_{x}+g \zeta_{x}=-p_{1, x} / \rho,
$$

where $\rho$ is the density of the fluid. 


\section{A.N. Golubiatnikov}

It is very helpful to introduce a mass of part of the layer $m$ which is calculated, for example, from a movable wall (piston) as an independent Lagrangian variable entering into the function $x(t, m)$. So, the first equation in Eqs. (4) gives $\rho(\zeta+h)=$ $1 / x_{m}$ and $v=x_{t}$. As a result, we get

$$
\rho x_{t t}+\left(g /\left(2 x_{m}^{2}\right)\right)_{m}=\rho g h_{x}-p_{1, x} .
$$

Eq. (5) has the form of the Euler equation with a Lagrangian

$$
\Lambda=\frac{\rho x_{t}^{2}}{2}-\frac{g}{2 x_{m}}+\rho g h(x, t)-p_{1}(x, t),
$$

which will be used later to solve the problem with a weak discontinuity.

The conditions for strong discontinuities (discontinuities of the first derivatives of the law of motion $x(t, m)$ ) in the absence of concentrated inflows of mass, momentum and energy follow from the form of $\Lambda[3]$. Let $t=T(m)$ be the time of motion in the mass of discontinuity. Let us introduce a shock time $\tau=t-T(\mathrm{~m})$ that is equal to zero on the discontinuity. Thus, in the variables $\tau, m$ we have

$$
x_{t}=x_{\tau}, \quad x_{m}(t, m)=x_{m}(\tau, m)-T^{\prime}(m) x_{\tau} .
$$

It should be mentioned that with these variables, the operation of differentiation with respect to $m$ on the discontinuity saves the continuity of the differentiated function.

With a strong discontinuity, we have

$$
[x]=0, \quad\left[\Lambda_{x_{\tau}}\right]=0 .
$$

In addition to that conditions, one more inequality must be introduced which is determined by the energy dissipation in the shallow water theory approximation [1],

$$
\left[x_{\tau} \Lambda_{x_{\tau}}-\Lambda\right]_{0}^{1} \leq 0 .
$$

The zero state corresponds to the state before the discontinuity and the unity state corresponds to the state after the discontinuity. The function $T(m)$ on the strong discontinuity is also unknown. In the case of weak discontinuity which always propagates with a characteristic velocity, the motion of discontinuity against the known background is also known. In this case, there is a discontinuity of the second derivations or of derivations of higher order of the function $x(t, m)$, and the values of these derivatives are defined by the motion equations and their differential continuations (by the transport equations [5]).

2. Discontinuities of small amplitude. We consider the class of solutions to Eqs. (5) with the weak discontinuity created by a piston's analytical motion of the form

$$
x_{p}(t)=x_{0}(0)+\alpha_{2} t^{2} / 2+\alpha_{3} t^{3} / 6+\ldots
$$

For simplicity, we restrict our consideration to the case of discontinuity motion against the known static backgroud $x_{0}(m)$ (at $h(x)$ and $p_{1}(x)$ ).

In [6], in the theory of ideal magnetohydrodynamics, a method of solution of one-dimensional problems with weak discontinuity which moves against an arbitrary background has been developed. In this paper, this method is presented in a more general Lagrangian form. The notations $v=x_{\tau}$ and $w=x_{m}(\tau, m)$ are used.

The definition of the background sound speed $1 / T_{0}^{\prime}$ is $\Lambda_{v v}^{0}=0$. There are $\Lambda_{v w}^{0}>0$ and $\Lambda_{v v v}^{0}<0$ for the Lagrangian Eq. (6). Let $\alpha_{2} \neq 0$. Then the solution 
of the first transport equation (Riccati's equation [5]) defines the acceleration of the fluid on the discontinuity

$$
v_{\tau}=\frac{1}{\left(\Lambda_{v w}^{0}\right)^{1 / 2}}\left(C_{2}+\int_{0}^{m} \frac{\Lambda_{v v v}^{0}}{2\left(\Lambda_{v w}^{0}\right)^{3 / 2}} \mathrm{~d} m\right)^{-1}
$$

All derivatives of the background are calculated provided $v_{0}=0$.

It should be mentioned that the value of acceleration has an order of unity, but the velocity is exactly zero. All next derivatives with respect to $\tau$ of the law of motion are defined by linear equations in quadratures. The constant $C_{2}$ is proportional to the initial acceleration of the piston $\alpha_{2} \neq 0$. If $\alpha_{2}<0$, the acceleration of the fluid is always negative. If $\alpha_{2}>0$, there is a possibility for the acceleration to take an infinite value in a finite time (overturning of weak discontinuity). The case of $\alpha_{2}=0$ corresponds to a special solution of the Riccati's equation $v_{\tau}=0$.

Let us consider now the following situation: there is a small, in relation to the background speed of sound, initial velocity of the piston $\alpha_{1}>0$ that creates a weak shock wave. We linearize the second condition in the discontinuity (8) and find a correction to the value of $T_{0}^{\prime}$ which corresponds to the velocity of the shock wave,

$$
\delta T^{\prime}=-\frac{\Lambda_{v v v}^{0}[v]}{2 \Lambda_{v v T^{\prime}}^{0}}
$$

Then we linearize the equation of motion in relation to the small velocity $v$ in the discontinuity, solve the correspond linear differential equation and, using Eq. (11), find

$$
v=\frac{C_{1}}{\left(\Lambda_{v w}^{0}\right)^{1 / 2}}\left|1+\int_{0}^{m} \frac{\Lambda_{v v v}^{0} \mathrm{~d} m}{2 C_{2}\left(\Lambda_{v w}^{0}\right)^{3 / 2}}\right|^{-1 / 2} .
$$

The constant $C_{1}$ could be expressed via $\alpha_{1}$.

Formula (13) shows that if $C_{2}$ is positive, along with the overturning of the weak discontinuity when $v_{\tau} \rightarrow \infty$, the velocity $v$ for any value of $C_{1}>0$ grows unboundedly, too. This evidences that the shock wave becomes strong and the represented theory is not applicable on the merits.

In the case of equillibrium, the second equation in (4) yields

$$
\rho g \zeta_{0}+p_{1}=C_{0}, \quad w_{0}=\frac{g}{C_{0}-p_{1}+\rho g h} .
$$

Calculating the derivatives of the Lagrangian Eq. (6) yields

$$
\Lambda_{v w}^{0}=\frac{g T_{0}^{\prime}}{\rho w^{3}}, \quad \Lambda_{v v v}^{0}=-\frac{3 g\left(T_{0}^{\prime}\right)^{3}}{\rho w_{0}^{4}}, \quad T_{0}^{\prime}=\left(\frac{\rho w_{0}^{3}}{g}\right)^{1 / 2}
$$

It is convenient to procede to the variable $x$, taking into account that $w \mathrm{~d} m=$ $\mathrm{d} x$. Then for the acceleration of the fluid in the weak discontinuity, we get

$$
v_{\tau}=\frac{g}{\rho} w_{0}^{3 / 4}\left(C_{2}-\frac{3}{2} \int_{x_{p}(t)}^{x_{0}(t)} w_{0}^{7 / 4} \mathrm{~d} x\right)^{-1}
$$




\section{A.N. Golubiatnikov}

and for the velocity on the weak shock wave we get

$$
v=C_{1}(\rho / g)^{1 / 4} w_{0}^{3 / 4}\left|1-\frac{3}{2 C_{2}} \int_{x_{p}(t)}^{x_{0}(t)} w_{0}^{7 / 4} \mathrm{~d} x\right|^{-1 / 2}
$$

where $x_{0}(t)$ corresponds to the motion of the background characteristic $t=T_{0}(\mathrm{~m})$.

The analysis of the formulae has shown that a singularity can appear which is determined by the equality to zero of the denominator of the specific volume $w_{0}$ (14) when the discontinuity tends to the zero thickness of the layer $\zeta_{0}+h=0$. It is possible only when the acceleration of the piston is negative. In this case, we can trace the attenuation process of the shock wave $[6,7]$. However, if the acceleration is positive before this situation, the overturning of the weak discontinuity occurs.

3. Acknowledgements. The work was provided with partial financial support of the Russian Foundation for Basic Research (Nos. 15-01-00361, 17-0100037).

\section{References}

[1] L.D. Landau, E.M. Lifshitz. Fluid Mechanics (Pergamon Press, 1987).

[2] S. Leibovich, A.R. Seebass. Examples of dissipative and dispersive systems leading to the Burgers and the Korteweg de Vries Equations. Collection of Articles "Nonlinear Waves" (Cornell University Press, 1974), pp. 103-138.

[3] L.I.Sedov. A Course in Continuum Mechanics (Vols. I-IV, WoltersNoordhoff Publishing, 1971).

[4] L.D. Landau, E.M. Lifshitz. Electrodynamics of Continuous Media (Pergamon Press, 1984).

[5] B.L. Rozhdestvenskit, N.N. Yanenko. Systems of Quasilinear Equations and Their Applications to Gas Dynamics (Providence-Rhode Island: S.n., 1983. American Mathematical Society. Translations of Mathematical Monographs. Volume 55).

[6] A.N. Golubyatnikov, S.D. Kovalevskaya. Propagation of discontinuities against a static background. Fluid Dynamics, vol. 52 (2017), issue 2, pp. 321328.

[7] G.G. Chernyi. Gas Dynamics (Moscow, Nauka Publ. House, 1988) (in Russian).

Received 27.12.2017 